\title{
The star RR Lyr and the Cepheid variables in the era of the space photometry revolution
}

\author{
Ennio Poretti ${ }^{1,2,3,4}$, Jean-François Le Borgne ${ }^{2,3,4}$, and Alain Klotz ${ }^{2,3,4}$ \\ 1 INAF-Osservatorio Astronomico di Brera, Merate, Italy \\ 2 Université de Toulouse, UPS-OMP, IRAP, Toulouse, France \\ 3 Groupe Européen d'Observations Stellaires (GEOS), Bailleau l'Evéque, France \\ ${ }^{4}$ CNRS, IRAP, Toulouse, France
}

\begin{abstract}
The long-term behaviours of the pulsation and Blazhko periods of RR Lyr are investigated by means of Kepler and ground-based observations. The difficulties in detecting additional modes in the Cepheids monitored with CoRoT are discussed.
\end{abstract}

\section{RR Lyr}

RR Lyr=KIC7198959 was included in the field-of-view of the Kepler space telescope [2] and highprecision, continuous observations could be secured. Well before the launch of Kepler, we decided to devise small, autonomous, and transportable photometric instruments to make the ground-based survey of the modulations in amplitude and phase (Blazhko effect) of RR Lyr as effective as possible. The instruments are composed of a commercial equatorial mount (Sky-Watcher HEQ5 Pro Goto), an AUDINE CCD camera (512x768 kaf400 chip) and a photographic $135-\mathrm{mm}$ focal, $\mathrm{f} / 2.8$ lens with a field of view of $2^{\circ} \times 3^{\circ}$ (Fig. 1). We gave them the nickname VTTs for "Very Tiny Telescopes".

The GEOS (Groupe Européen d'Observations Stellaires) RR Lyr database [5] is continuously updated by inserting all the published maxima of RR Lyr variables. RR Lyr itself is among them ${ }^{1}$. Therefore, we could analyse [6] all the observed maxima since 1899 in order to have a clear picture of the behaviours of the pulsation period $P_{0}$ and of the Blazhko effect, noticed since 1916. We could identify two states defined as pulsation over a "long" primary period $\left(P_{0}>0.56684 \mathrm{~d}\right)$ and over a "short" one $\left(P_{0}<0.56682 \mathrm{~d}\right)$. These states alternate with intervals of $13-16 \mathrm{yr}$, and are well defined since 1943. We also provided homogeneous determinations of the Blazhko period $P_{B}$ in several time intervals and we studied how it changed while the two states alternated. We could clearly establish how the Blazhko period had just one sudden decrease from $40.8 \mathrm{~d}$ to $39.0 \mathrm{~d}$ in 1975 . The variations of the pulsation and Blazhko periods are completely decoupled (Fig. 2).

Moreover, the combination of Kepler and VTT data recorded the vanishing of the Blazhko effect. The space telescope continuously monitored the monotonic long-term decrease, proving that smallscale modulations, lasting from 2 to $4 P_{B}$, are also visible in the O-C (observed minus calculated times of maximum brightness) values. The VTT data are now securing the RR Lyr monitoring after that Kepler had to be pointed in another direction. In particular, Fig. 2 shows how the large sine-shaped curve of the $\mathrm{O}-\mathrm{C}$ values observed in 2008 turned out to be an almost straight line in 2014. The Blazhko nature of the light variability of RR Lyr is hard to detect by looking only to the maxima collected in the year 2014.

\footnotetext{
1 http://rr-lyr.irap.omp.eu/dbrr/dbrr-V1.0_08.php?RR\%20Lyr)

This is an Open Access article distributed under the terms of the Creative Commons Attribution License 4.0, which permits unrestricted use, distribution, and reproduction in any medium, provided the original work is properly cited.
} 


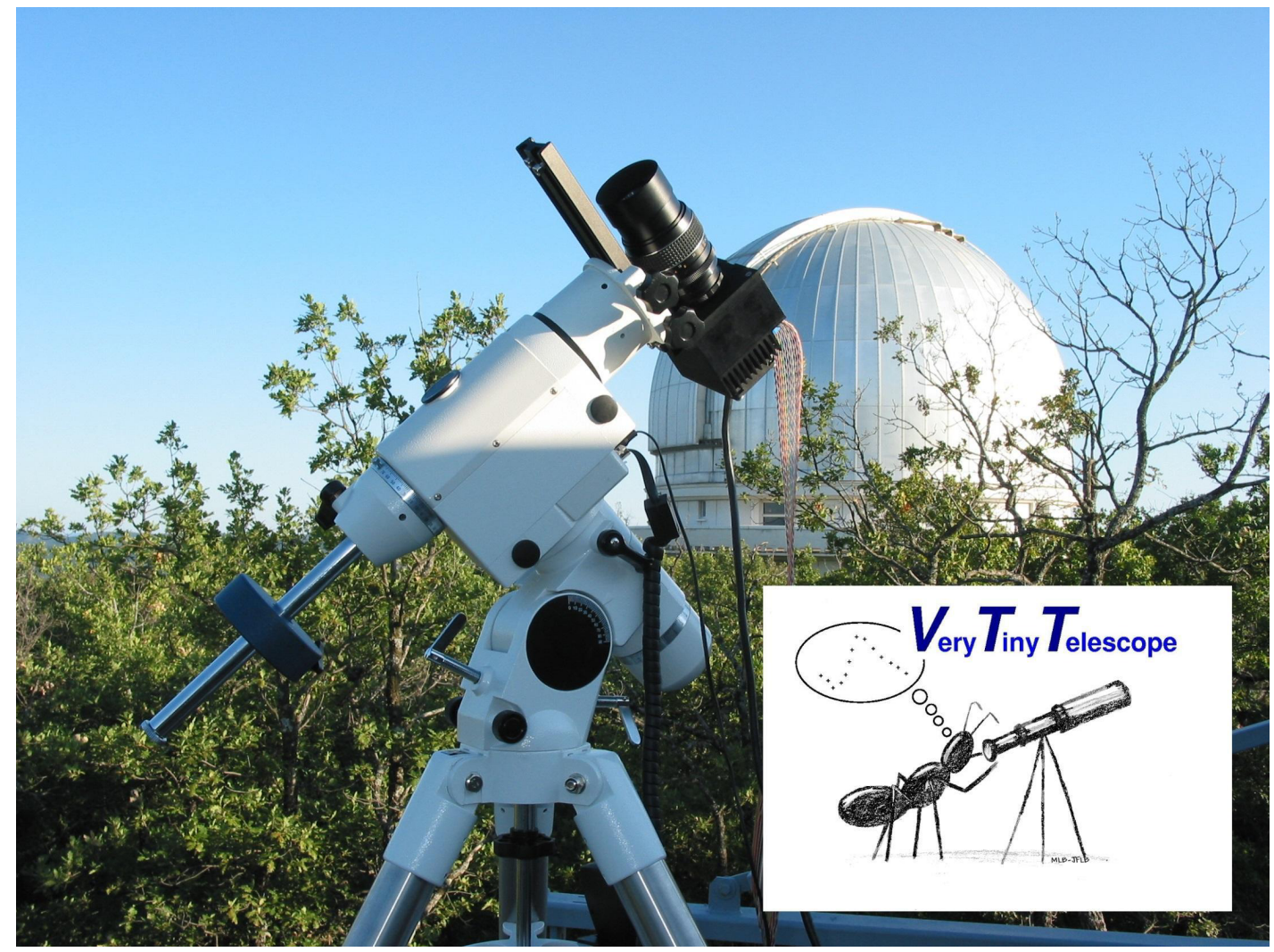

Fig. 1. The Very Tiny Telescope (VTT). In the background the dome of the 1.93-m telescope at the Observatoire de Haute-Provence.

\section{Cepheids}

Though nonradial modes were already detected in classical pulsators from ground-based surveys, it has been the space revolution that provided us clear cases of the excitation of nonradial modes in highamplitude $\delta$ Sct stars and RR Lyr variables. In this revolutionary context, Cepheids are playing the role of the conservative party, since additional modes are very rare in their light curves. Low amplitude frequencies were found in OGLE-II stars [7], more specifically secondary peaks in proximity to the dominant first overtone radial mode. The stars could be both single-periodic first-overtone Cepheids and double-mode ones. Intriguing cases are also those of Cepheids showing one secondary mode with frequency much above that of the radial mode. The situation did not change after the Kepler pointing in the Lyra-Cygnus direction: there was only one Cepheid in the field-of-view, i.e., V1154 Cyg. The detailed analysis of the data acquired in the first 600 days (i.e., about 120 cycles) of the mission detected significant cycle-to-cycle fluctuations [3]. A very slight correlation was also found between the Fourier parameters and the $\mathrm{O}-\mathrm{C}$ values, suggesting that the $\mathrm{O}-\mathrm{C}$ variations (up to $30 \mathrm{~min}$ ) might be due to instabilities in the light curve shape. This correlation was strengthened by the analysis of the Kepler measurements up to Q17 [4].

Recently we re-investigated all the variables observed by CoRoT [1] in the exoplanetary field. We were able to detect eight Cepheids. The most important case was that of CoRoT 0223989566, that resulted to be an unusual triple-mode Cepheid located very probably in the "outer arm" of the Milky Way [8]. Two other Cepheids were observed in Long Runs and therefore we could perform a detailed frequency analysis to investigate the excitation of additional modes. The case of CoRoT 0102618121 was particularly relevant in this context. After subtracting the main oscillation, the residuals obtained both in the LRa01 (131 d long) and in the LRa06 (76 d) data revealed a possible peak at $0.488 \mathrm{~d}^{-1}$. 

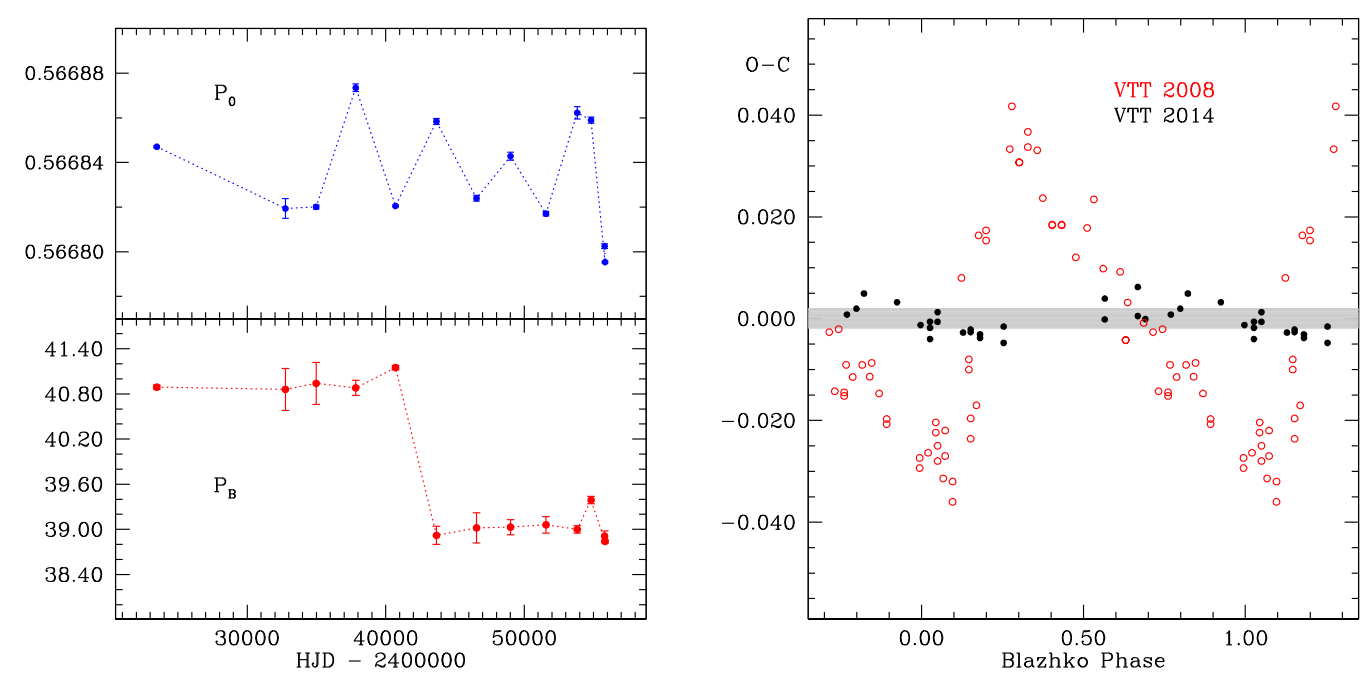

Fig. 2. Measure units of the ordinates are days. Left panel: decoupled variations of the pulsation period $P_{0}$ (top) and of the Blazhko period $P_{B}$ (bottom) from 1920 to 2013. Right panel: the vanishing amplitude of the O-C values from 2008 ( $0.062 \mathrm{~d}$, red empty circles) to 2014 ( $0.002 \mathrm{~d}$, black filled circles). The gray band delimites the $\pm 1 \sigma$ interval around the mean of the O-C values obtained in 2014.

However, when computing the residuals in another way, i.e., cycle-by-cycle, this peak disappeared from the power spectra of both runs. Indeed, the light curve obtained from plotting the cycle-by-cycle residuals is completely flat and very different from that from the fit of the entire dataset. We also notice that we did not detect any linear combination between the main pulsation frequency and the $0.488 \mathrm{~d}^{-1}$ one: this fact does not support the identification of the latter term as an additional mode. Our working hypothesis is that the light curve is affected by very small jumps and drifts, leaving some traces of the large amplitude, main oscillation after removing a solution with fixed amplitudes, phases and frequencies from the data.

As an example of these subtle instrumental effects, let us consider the light curves obtained by CoRoT in the $r_{C}, g_{C}$, and $b_{C}$ colours. The most relevant perturbation occurred around JD 2454487.5 (Fig. 3): a sort of blurring of the light curve that lasted for about $0.5 \mathrm{~d}$. We can suppose that the star's image jumped all around due to a sudden increase of the satellite jitter. As a result, the flux subdivision changed: the counts decreased in the $b_{C}$ and $g_{C}$ curves, while increased in the $r_{C}$ one. However, the total counts did not change significantly and the white light curve perfectly describes the star's variability. A different event occurred one cycle later. The $b_{C}$ curve shows a sudden jump, followed by a decline to the expected flux level. This event was not recorded in $g_{C}$ and $r_{C}$ colours (zoom-in in the inserted boxes). Probably a cosmic ray hit a pixel in the $b_{C}$ submask. Therefore, the curve in white light shows the event on a reduced scale with respect to the $b_{C}$ one. These abrupt changes are hard to be noticed by eye and the automatic removal is quite dangerous. Indeed, rapid variations are what we are willing to put in evidence with the goal to detect possible planetary transits. On the contrary, the cycle-to-cycle fits minimize such effects leaving a light curve cleaner than that of the fit of the entire dataset. We cannot rule out that we are actually observing small cycle-to-cycle variations due to some physical mechanism not strictly periodic, as granulation or stellar activity. Further analyses are in progress [9]. 


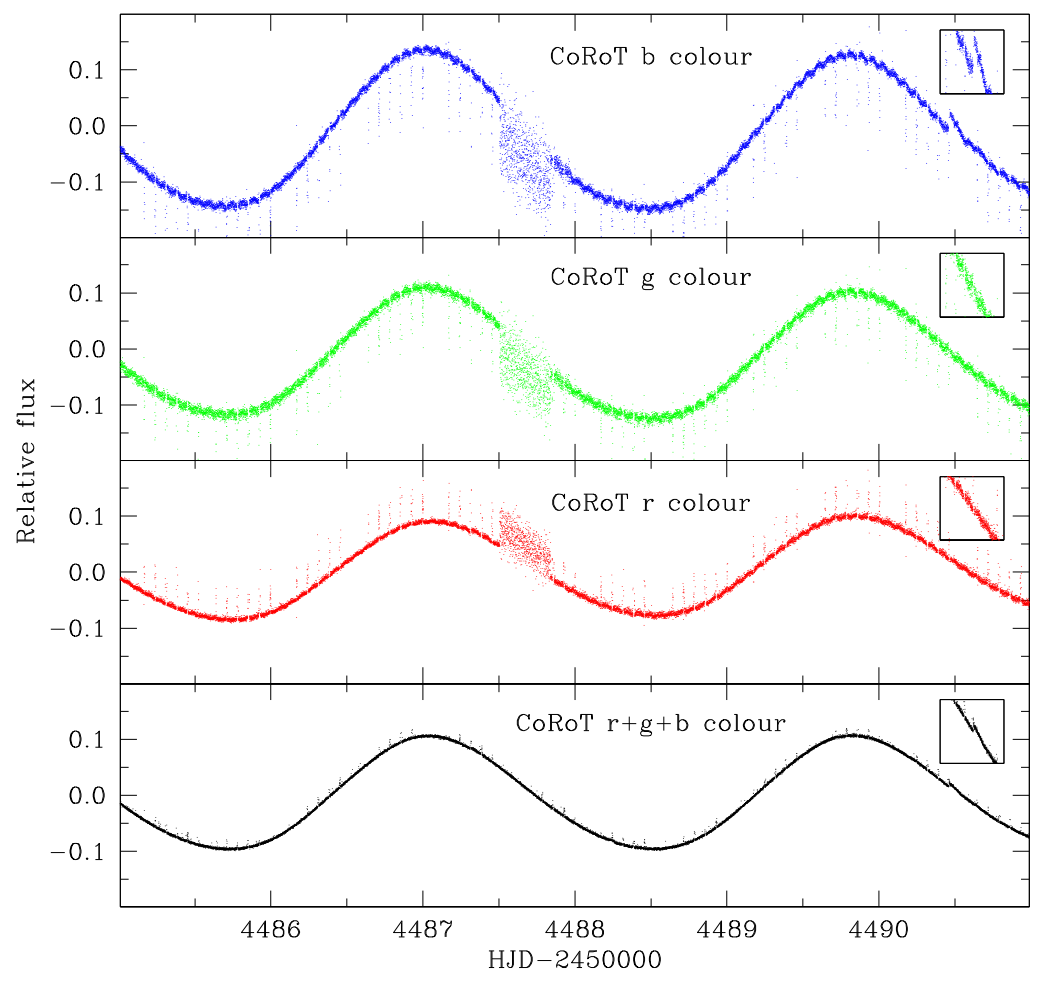

Fig. 3. The CoRoT light curves in $r_{C}, g_{C}, b_{C}$ and white colours reflect in different ways the effect of the satellite jitter around JD 2454487.5. The jump around JD 2454490.5 (inserted boxes) was observed in the $b_{C}$-light, but not in the $r_{C}$ and $g_{C}$ ones.

EP acknowledges Observatoire Midi-Pyrénées for the two-month grant allocated between 2014 May and July, allowing him to spend a very fruitful stage at the Institut de Recherche en Astrophysique et Planétologie in Toulouse, France.

\section{References}

1. Baglin, A., Auvergne, M., Barge, P., et al., in The CoRoT Mission, Pre-Launch Status, Stellar Seismology and Planet Finding, ESA SP-1306, (ESA, Nordwijk), (2006), 33

2. Borucki, W. J., Koch, D., Basri, G., et al., Science, 327, (2010), 977

3. Derekas, A., Szabó, Gy. M., Berdnikov, L., et al., MNRAS, 425, (2012), 1312

4. Kanev, E., et al., these proceedings, (2015)

5. Le Borgne, J.F., Paschke, A., Vandenbroere, J., et al., A\&A, 476, (2007), 307

6. Le Borgne, J.F., Poretti, E., Klotz, A., et al., MNRAS, 441, (2014), 1435

7. Moskalik, P., Precision Asteroseismology, IAU Symp. Vol. 301 (Cambridge: Cambridge Univ. Press), (2014), 249

8. Poretti E., Baglin A., Weiss W.W., ApJ, 795, (2014), L36

9. Poretti E., et al., in preparation 